Available online on 15.07.2018 at http://jddtonline.info

Journal of Drug Delivery and Therapeutics

Open Access to Pharmaceutical and Medical Research

(c) 2011-18, publisher and licensee JDDT, This is an Open Access article which permits unrestricted

non-commercial use, provided the original work is properly cited

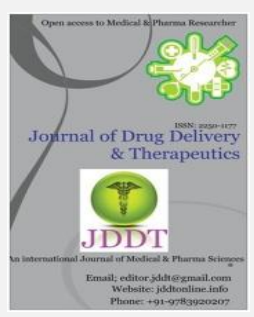

Open $\odot$ Access

Research Article

\title{
Study on Anti-inflammatory and Analgesic Effect of FangXiangTongLuo-cervical Plaster
}

Linfeng $\mathrm{Li}^{1}$, Ruomei Che ${ }^{1}$, Siqi Li ${ }^{1}$, Dandan $\mathrm{Du}^{1}$, Dandan Han ${ }^{2}$, Yutong $\mathrm{Li}^{1}$, Chen Liu ${ }^{1}$, Nan Hao ${ }^{1}$, Haonan $\mathrm{Wu}^{1}$, Shihui Sun ${ }^{1,}$ *

${ }^{1}$ Department of Traditional Chinese Medicine, Hebei University, Baoding, China

${ }^{2}$ Department of Clinical Medicine, Hebei University, Baoding, China

\section{ABSTRACT}

Objective: To study the anti-inflammatoryandanalgesic effects of FangXiangTongLuo-cervical Plaster. Methods: Preparation of FangXiangTongLuo cervical ointment. Using the model of the mouse ear swelling inflammation which is caused by xylene. The analgesic mode of hot-plate method and acetic acid wrinkle method, in order to study the anti - inflammatory and analgesic effects of FangXiangTongLuo-cervical Plaster. Result: The results showed that Fangxiangtongluo cervical plaster can effectively reduce the number of writhing in acetic acid-induced mice and increase the rate of writhing inhibition and analgesic percentage $(p<0.01)$. In the hot plate experiment, Fangxiangtongluo cervical plaster can effectively improve the pain threshold of mice. With the increase in medication time, it can also significantly extend the time of the first mouse licking foot, thereby increasing the pain threshold of the mice. At the same time, Fangxiangtongluo cervical plaster can also significantly inhibit the degree of swelling of the mouse auricle caused by xylene, reducing the exudation of inflammatory substances in the body of mice. Conclusion: Fangxiangtongluo cervical plaster has significant anti-inflammatory and analgesic effects.

Keywords: FangXiangTongluo; cervical Plaster; anti-inflammation; analgesia

Article Info: Received 30 April, 2018; Review Completed 06 June 2018; Accepted 09 June 2018; Available online 15 July 2018

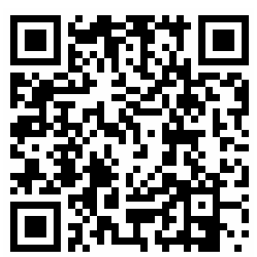

Cite this article as:

Li L, Che R, Li S, Du D, Han D, Li Y, Liu C, Hao N, Wu H, Sun S, Study on Anti-inflammatory and Analgesic Effect of FangXiangTongLuo-cervical Plaster, Journal of Drug Delivery and Therapeutics. 2018; 8(4):223-228

DOI: http://dx.doi.org/10.22270/jddt.v8i4.1777

*Address for Correspondence:

Shihui Sun, Department of Traditional Chinese Medicine, Hebei University, Baoding, China, E-mail: 121826311@qq.com

This article is the result of the scientific research project of Hebei provincial administration of traditional Chinese medicine (project no. 2017219). 


\section{INTRODUCTION}

With the accelerated pace of social life, the popularity of electronic products such as computers and mobile phones, improper fixation in a long time causes cervical muscle spasm and inflammation, over time, the physiological curvature of the cervical spine changes, and cervical facet joint disorders, as a result, the incidence of cervical spondylosis is significantly increased ${ }^{1}$. If the condition progresses further, there may be obvious movement loss, regular gait difficulty and balance dysfunction, it seriously affects the quality of life of the patients ${ }^{2}$. The recurrence of cervical spondylosis and the gradual worsening of the disease have caused great physical and mental pain to the patient and reduced their work and quality of life $^{3}$. Therefore, we put forward FangXiangTongLuo-cervical Plaster, It can be used for long term cervical vertebra health care, local treatment to relieve inflammation and relieve pain. In this study, animal experiments were conducted to verify the anti-inflammatory and analgesic effects of FangXiangTongLuo-cervical Plaster formula.

\section{MATERIALS AND GROUPING}

\section{Animals}

80 SPF Kunming mice, both male and female, weighing 18-22 g, provided by Experimental Animal Center of Hebei Medical University, the license number is SCXK2013-1003.

\section{Drugs and reagents}

The experimental drugs were divided into improved group, traditional group, western medicine group and control group.

Improved group: Astragalus 30g, Divaricate Saposhniovia Root 30g, Atractylodes 15g, Ephedra 15g, Kudzu 15g, Rhizoma et radix notopterygii $15 \mathrm{~g}$, Gastrodia $30 \mathrm{~g}$, Cinnamon $10 \mathrm{~g}$, Dry ginger 10g, Herb of Tuberculate Speranskia 30g, Common Clubmoss Herb30g, Mulberry branch 30g, Rhubarb 30g, Acorus calamus 10g, Lilac 10g, Cortex Cinnamon $10 \mathrm{~g}$, fresh rose $60 \mathrm{~g}$, fresh lily $60 \mathrm{~g}$, fresh chrysanthemum $60 \mathrm{~g}$.

Traditional group: Astragalus 30g, Divaricate
Saposhniovia Root 30g, Atractylodes 15g, Ephedra 15g, Kudzu 15g, Rhizoma et radix notopterygii 15g, Gastrodia $30 \mathrm{~g}$, Cinnamon 10g, Dry ginger $10 \mathrm{~g}$, Herb of Tuberculate Speranskia 30g, Common Clubmoss Herb30g, Mulberry branch 30g, Rhubarb 30g, Lilac 10g, Cortex Cinnamon 10g, Radix Aconiti 15g, Radix Aconiti Kusnezoffii 15g, Rhizoma Typhoni 15g, Asarum 10g, Borneol 10g. The processing method is the same as the improved group.

Western medicine group: Diclofenac sodium patch.

Control group: blank matrix group.

Reagents: Anhydrous ethanol solution, citric acid, 0.6\% acetic acid solution, glycerol, polyethylene glycol 400 (PEG400), sodium hydroxymethyl cellulose, aluminum hydroxyacetate, sodium polyacrylate, povidone, xylene.

\section{Instrument}

HH-S type constant temperature water bath(Jintan Zhengji Instrument Co., Ltd.), BS1103 Sartorius electronic Balance (Beijing Sartorius Balance Co., Ltd.), KQ-250B Ultrasonic Cleaner (Kunshan Ultrasonic Instrument Co., Ltd.), GJ8402 Hot Plate Tester (Zhejiang Haining Baishi Electric Medical Instrument Factory), INS500078-5 size mouse ear perforator (Beijing Chuang Bo Biotechnology Co., Ltd.), YUYAN animal shaving machine (Yuyan Scientific Instrument Co., Ltd.).

\section{Animal grouping}

The experimental animals were randomly divided into four groups as required: blank matrix negative control group, traditional group, modified group, western medicine positive control group. Each group of experimental animals was administered according to the corresponding experimental method, and appropriate treatment of the administration site to avoid foraging.

\section{Experimental section}

\section{Preparation of drugs}

The volatile oil was extracted from the traditional Chinese medicine by soxhlet extractio. The remaining liquid is heated, concentrated and dried into extremely fine powder by water bath. Babuso base used: glycerol-water-ethanol-polyethylene glycol 400

This article is the result of the scientific research project of Hebei provincial administration of traditional Chinese medicine (project no. 2017219). 
(PEG400)-aluminum glyceride-citric acid-sodium carboxymethyl cellulose-sodium polyacrylate-povidone, according to $2: 8: 0.8: 0.4: 0.07: 0.15: 0.1: 0.5: 0.5$, the fine material and the matrix are mixed in a certain proportion, then amixture of fine material and substrate is mixed to make paste, and the thickness of the paste is $2.80 \mathrm{~mm}$.

\section{Analgesic experiment (hot plate method)}

Forty female mice, 10 in each group, were randomly divided into the modified group, the traditional group, the western medicine group, and the control group. First determine the pre-pain threshold, The mice were placed on a constant temperature hot plate at $55^{\circ} \mathrm{C} \pm 0.5^{\circ} \mathrm{C}$, each mouse was recorded as a pain threshold from the time it was placed on the hot plate to the foot after licking, select non responders in 30 s and leapfrog in $5 \mathrm{~S}$ as unqualified mice. Repeat measurement once, the mean value of the two measurements was taken as the pre-administration pain threshold in mice. Secondly, four kinds of experimental drugs were applied to mouse hind feet. After the administration (after smearing on the mouse foot pad), the pain threshold was measured at 15, 30, and $60 \mathrm{~min}$. If there is no response to $60 \mathrm{~s}$ on the hot plate, the mice should be removed immediately and calculated according to $60 \mathrm{~s}^{4}$, calculation of the increase rate of pain threshold in mice.

\section{Writhing experiment}

40 mice (male and female) and 10 rats in each group, were randomly divided into 4 groups: the improved group, the traditional group, the western medicine group and the control group. In the abdominal hair removal of mice
$(1 \mathrm{~cm} * 1 \mathrm{~cm})$, four experimental drugs were applied to the depilation area, once a day for 3 consecutive days. After the last administration of $2 \mathrm{~h}$, mice in each group were given $0.6 \%$ acetic acid $0.1 \mathrm{ml} / 10 \mathrm{~g}$ respectively. The number of writhing appeared in $15 \mathrm{~min}$ after administration of mice was determined ${ }^{5}$, and the inhibition rate was calculated.

\section{Anti-inflammatory test (Xylene-induced mouse ear swelling method)}

40 mice (male and female) and 10 rats in each group, were randomly divided into 4 groups: the improved group, the traditional group, the western medicine group and the control group. Apply each group of drugs evenly on both sides of the left ear of the mouse. Apply $0.1 \mathrm{ml}$ xylene to each side of the left ear of the mouse 30 minutes after administration, and inhale only inflammation, no treatment is performed on the right ear. After 1 hour, the animals were sacrificed, and the two ears of the mice were cut along the baseline of the auricle. The ear was dropped on the same site of both ears with a hole punch of $8 \mathrm{~mm}$, weighed, and the ear swelling degree and inhibition rate were calculated ${ }^{6}$.

[degree of swelling $(\mathrm{mg})=$ right ear weight - left ear weight]

\section{Statistical method}

The SPSS 22 statistical software was used for data processing, and the measured data were expressed as mean \pm standard deviation ( $\overline{\mathrm{x}} \pm \mathrm{s})$, and one-way ANOVA(One-way ANO-VA) was used for comparison between groups . 


\section{RESULT}

\section{Experimental results of hot plate}

Table 1 : experimental data of hot plate analgesia $(\overline{\mathrm{x}} \pm \mathrm{s})$ in mice

\begin{tabular}{|c|c|c|c|c|c|}
\hline \multirow[t]{2}{*}{ Groups } & \multirow[t]{2}{*}{$\mathrm{n}$} & \multirow{2}{*}{$\begin{array}{c}\text { Pain threshold } \\
\text { before } \\
\text { administration }\end{array}$} & \multicolumn{3}{|c|}{ Pain threshold after administration } \\
\hline & & & $15 \mathrm{~min}$ & $30 \mathrm{~min}$ & $60 \mathrm{~min}$ \\
\hline the improved group & 10 & $21.94 \pm 2.54$ & $26.02 \pm 1.68^{\mathrm{ac}}$ & $34.20 \pm 2.23^{\mathrm{ac}}$ & $40.18 \pm 2.04^{\mathrm{a}}$ \\
\hline the traditional group & 10 & $22.40 \pm 2.12$ & $29.21 \pm 1.70^{\mathrm{ab}}$ & $37.29 \pm 1.80^{\mathrm{ab}}$ & $41.70 \pm 2.80^{\mathrm{ab}}$ \\
\hline the western medicine group & 10 & $21.89 \pm 2.22$ & $27.72 \pm 2.75^{\mathrm{ac}}$ & $35.22 \pm 2.62^{\mathrm{ac}}$ & $39.39 \pm 1.75^{\mathrm{ac}}$ \\
\hline the control group & 10 & $21.31 \pm 2.22$ & $23.65 \pm 1.17^{\mathrm{bc}}$ & $24.40 \pm 2.03^{\mathrm{bc}}$ & $25.60 \pm 1.76^{\mathrm{bc}}$ \\
\hline
\end{tabular}

Note: Compared with the control group, ${ }^{a} \mathrm{P}<0.05$; compared with the western medicine group, ${ }^{b} \mathrm{P}<0.05$; compared with the traditional group, ${ }^{\mathrm{c}} \mathrm{P}<0.05$.

Statistical analysis showed that there was no significant difference between the experimental groups and the control group before the administration $(\mathrm{P}>0.05)$, and the difference was not statistically significant. At 15 minutes after administration, there was a significant difference between the experimental groups and the control group $(\mathrm{P}<0.05)$, and the difference was statistically significant. The comparison relationship between other groups is shown in the table.

\section{Acetic Acid Writhing Test Results}

Table 2: experimental data of mice writhing ( $\overline{\mathrm{x}} \pm \mathrm{s})$

\begin{tabular}{|l|c|c|}
\hline \multicolumn{1}{|c|}{ Groups } & $\mathrm{n}$ & $\begin{array}{c}\text { Number of } \\
\text { writhing (Times) }\end{array}$ \\
\hline the improved group & 10 & $14.10 \pm 1.20^{\mathrm{ac}}$ \\
\hline the traditional group & 10 & $11.00 \pm 1.70^{\mathrm{ab}}$ \\
\hline the western medicine group & 10 & $14.60 \pm 1.96^{\mathrm{ac}}$ \\
\hline the control group & 10 & $22.20 \pm 2.30^{\mathrm{bc}}$ \\
\hline
\end{tabular}

Note: Compared with the control group, ${ }^{\mathrm{a}} \mathrm{P}<0.05$; compared with the western medicine group, ${ }^{b} \mathrm{P}<0.05$; compared with the traditional group, ${ }^{\mathrm{c}} \mathrm{P}<0.05$.

Statistical analysis showed that there was a significant difference between the experimental groups and the control group after administration $(\mathrm{P}<0.05)$, and the difference was statistically significant. The comparison relationship between other groups is shown in the table.

\section{Ear swelling test results}

Table 3: the swelling degree of the auricle of the experimental group and the control group $(\overline{\mathrm{x}} \pm \mathrm{s})$

\begin{tabular}{|l|c|c|}
\hline \multicolumn{1}{|c|}{ Groups } & $\mathrm{n}$ & Swelling degree \\
\hline the improved group & 10 & $29.20 \pm 1.44^{\mathrm{ac}}$ \\
\hline the traditional group & 10 & $25.83 \pm 1.71^{\mathrm{ab}}$ \\
\hline the western medicine group & 10 & $28.58 \pm 1.86^{\mathrm{ac}}$ \\
\hline the control group & 10 & $30.80 \pm 2.19^{\mathrm{bc}}$ \\
\hline
\end{tabular}

Note: Compared with the control group, ${ }^{\mathrm{a}} \mathrm{P}<0.05$; compared with the western medicine group, ${ }^{\mathrm{b}} \mathrm{P}<0.05$; compared with the traditional group, ${ }^{\mathrm{c}} \mathrm{P}<0.05$.

Statistical analysis showed that there was a significant difference between the experimental groups and the control group after administration, $(\mathrm{P}<0.05)$, and the difference was statistically significant. The comparison relationship between other groups is shown in the table.

\section{DISCUSSION}

The pathogenesis of cervical spondylosis is not fully understood in modern medicine, most scholars believe that the pathogenesis is due to destabilization caused by cervical disc or cervical degeneration, stimulate the sympathetic plexus around the vertebral artery, causing vertebral artery spasm stenosis, and the other side decompensated, causing vertebral artery insufficiency, appearing corresponding symptoms ${ }^{7}$. The traditional

This article is the result of the scientific research project of Hebei provincial administration of traditional Chinese medicine (project no. 2017219). 
Chinese medicine believes that the production of cervical spondylosis is affected by the cold, damp heat and evil, the dysfunction of blood in the internal injuries, and the intense meridians in the neck. After injury, it is easy to cause dysfunction of ascending and descending turbidity, besides neck pain, it is accompanied by other symptoms of general malaise. The traditional Chinese medicine believe that cervical spondylosis is similar to some manifestations of "arthralgia syndrome" ${ }^{8}$. The ancient Chinese book "Bingyinmaizhi" mentioned: "the obstruction of the arthralgia, the obstruction of the meridians, the indifference of the meridians, or the pain of the attack, or the condensation of the joints, or the difficulty of shifting the hands and feet, so that the name is called arthralgia." And "Yilin corrections" also mentioned "shoulder pain, arm pain, lumbago, leg pain, or pain around the body, always known as arthralgia." Therefore, in view of the "arthralgia" of the cervical spondylosis, the aromatic drugs have been added to the original clinical application of the effective cervical cream formula for many years, replacing the poisonous medicinal herbs such as Aconitum, expecting the patients to treat the cervical spondylosis better by using the non-toxic green formula.

From the perspective of daily health care, this study explores the advantages of external treatment and preventive treatment of TCM, a new formula with the effect of lifting and reducing turbidity and aromatic resuscitation is developed, and a new type of Babu paste is made to make a new type of plaster for cervical spondylosis, which plays a role in reducing toxicity and increasing efficiency. This prescription is based on Yuping wind powder, Gegen Decoction and Yuzhen San, it integrates wind protection, solid table, spasmodic pain relief, ascending and descending and turbidity lowering functions. At the same time, we improved the compatibility of traditional Chinese medicine plaster, and tried to use aromatherapy to refresh the mind and relieve pain, in order to replace the traditional Chinese medicine plaster, such as Radix Aconiti Kudo and Strychni, which are highly toxic. And this study used Babu preparation, compared with the traditional external plaster, Babu preparation has the advantages of controlled dosage, large This article is the result of the scientific research project of Hebei provincial administration of traditional Chinese medicine (project no. 2017219). amount of drug loading, small absorption area, stable blood concentration, convenient use, repeated paste and good air permeability ${ }^{9}$. In conclusion, the FangXiangTongLuo-cervical Plaster has the smell of fresh flowers without traditional Chinese medicine, and can be used for a long time, it is hoped that the progress of cervical spondylosis can be delayed or blocked to improve the quality of life of high-risk groups, improve the efficiency of work and study, and avoid the risk of future surgery.

The experimental results show that the FangXiangTongLuo-cervical Plaster has obvious analgesic effect. In the hot plate test of mice, the improved group compared with the control group, $\mathrm{P}<0.05$, compared with the western medicine group and the traditional group, there was no significant difference $(\mathrm{P}>0.05)$. The pain threshold of mice was significantly increased, and the pain induced reaction of mice induced by hot Spurs was prolonged. In the writhing test of mice, there was a significant difference between the improved group and the control group $(\mathrm{P}<0.05)$, while there was no significant difference between the improved group and the Western medicine group $(\mathrm{P}>0.05)$, but the improved group was better than the western medicine group in inhibiting the number of twisting bodies caused by acetic acid in mice. The analysis of related data shows that after removing Aconitum and Aconiti Lateralis, the analgesic effect of FangXiangTongLuo-cervical Plaster was lower than that of the traditional plaster prescription group, but the efficacy was higher than that of the western medicine group and the control group, and the difference between the efficacy and the traditional group is less, indicating that the efficacy of aromatic collaterals played a great role. In the ear swelling test, the drugs of the western medicine group, the traditional groupand the improved group compared with the control group, all have a suppressive effect on the mouse ear swelling caused by xylene, but the improved group compared with the Western medicine group $(\mathrm{P}>0.05)$, improved group slightly lower than the traditional group swelling effect, indicating that the modified group has a certain swelling effect.

The formulation of the FangXiangTongLuo uses the 
fragrance of fresh flowers to replace traditional toxic materials such as Aconiti and Radix Aconiti Kusnezoffii, and its anti-inflammatory, analgesic, and swelling effects will be lower than the traditional formula is the result of the experimental expected range. The specific reasons are related to herbs, such as Radix Aconiti, Radix Aconiti ksii, which are replaced by fresh flowers, which have good functions of warming meridians, dredging collaterals, dispelling wind and removing dampness. FangXiangTongLuo-cervical Plaster uses the FangXiangTongluo formulation and the new Babu Dosage form, the effect of reducing toxicity and increasing efficiency was higher than that of the effective

\section{REFERENCES}

[1] Zhang Yu. Zhang Shi Yi Tong [M]. Beijing: People's Medical Publishing House, 2006; 12:140-155.

[2] Zhu Zhiwei, Lu Zhu Sun. Case control study on the influencing factors of cervical spondylosis in young and middle-aged people in Shiyan. Chinese Journal of social medicine, 2016; (01):31-34.

[3] Jia Lianshun. Modern cervical surgery. Shanghai: Shanghai Far East press, 1993. 161 169.

[4] Zhan Xiang Yi, Wang Wenping, Wang Yan, Gao Feng, Cao Qi Chen, Analgesic effect of Chinese medicine Zhitong Babu paste. Journal of Liaoning University of Traditional Chinese Medicine, 2011; (7):45-46

[5] Chen Xiaoye, Zhang Yupeng, Jiang Xiaoli, Wang Su, Wang Lifang, Zou Shijie, Ouyang Haiyan, Diao Zuobin, Pang Daben. A preliminary study on the cold and heat properties of the pain model of hot plate method of acetic acid torsional pain. Journal sodium dichlorate group for clinical application, and the effect of the difference with the traditional plaster group is controlled within a certain range. It shows that the new formula can realize the green environmental protection of the cervical plaster, and can also be used for the health care of the cervical spondylosis for a long time. It lays the foundation for the research of the special effect green cervical cream. However, the analgesic and anti-inflammatory components of the FangXiangTongLuo-cervical Plaster and its mechanism remain to be further studied, at the same time; the impact of the green environmental impact of this formula also deserves our in-depth exploration. of Zhejiang Chinese Medicine University, 2005; 29(4):46-51.

[6] Lv Jinfang Li Dongfeng division Wu Song Ning Kang Jian should be like the sea. Experimental study on the inhibitory effect of Eucommia ulmoides leaves and eucommia bark on auricle swelling in mice by different processing methods. Chinese medicine science and technology, 2006; (6):399-400.

[7] Wu Yaonan, Sun Zhi he, Cai Yongrui. Clinical analysis of Chinese medicine in treating cervical spondylosis of vertebral artery type. Chinese community doctor (medical profession), 2012; (20):201-202.

[8] Feng Fei, Jiang Ya Jun. Discussion on Chinese medicine treatment of cervical spondylosis. Journal of Zhejiang Chinese Medicine University, 2011; (06):881-883.

[9] Sun Hongchang. A preliminary study on the applicable advantages of Babu agent in Department of Dermatology. Technology wind, 2011; (22):55-56. 